\title{
Fe-Coordinated Carbon Nanozyme Dots as Peroxidase-like Nanozymes and MRI Contrast Agents
}

Ruixue Qinł, Yushuo Fengł, Dandan Ding, Lei Chen, Shi Li, Huaping Deng, Shileng Chen, Zhenxin Han, Wenjing Sun*, Hongmin Chen*

State Key Laboratory of Molecular Vaccinology and Molecular Diagnostics \&

Center for Molecular Imaging and Translational Medicine, School of Public Health, Xiamen University, Xiamen 361102, China

Corresponding Authors

*E-mail: sunwenjing1102@163.com ;

*E-mail: hchen@xmu.edu.cn

$\ddagger$ These authors contributed equally 
a

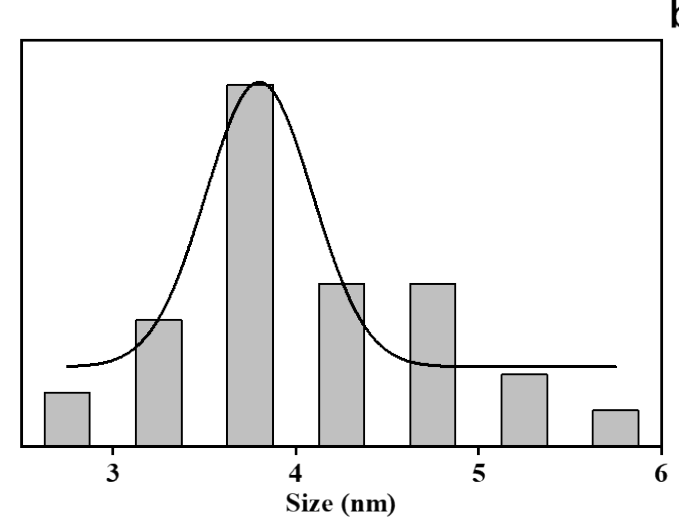

b

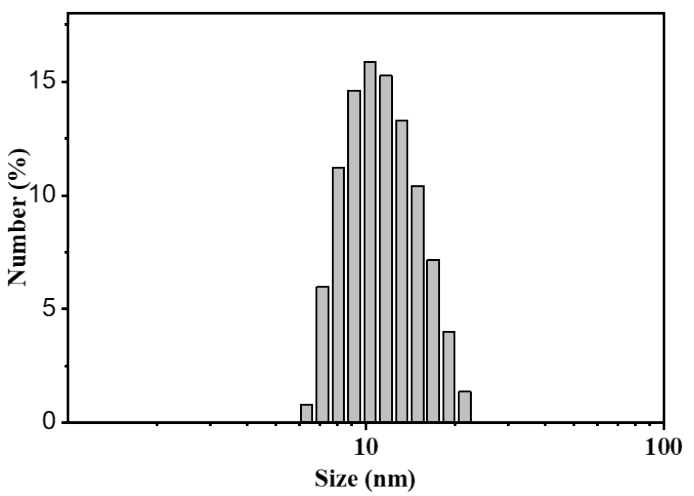

Figure S1. (a) The size of Fe-CDs measured from Figure 1a. (b) Dynamic light scattering of Fe-CDs. 

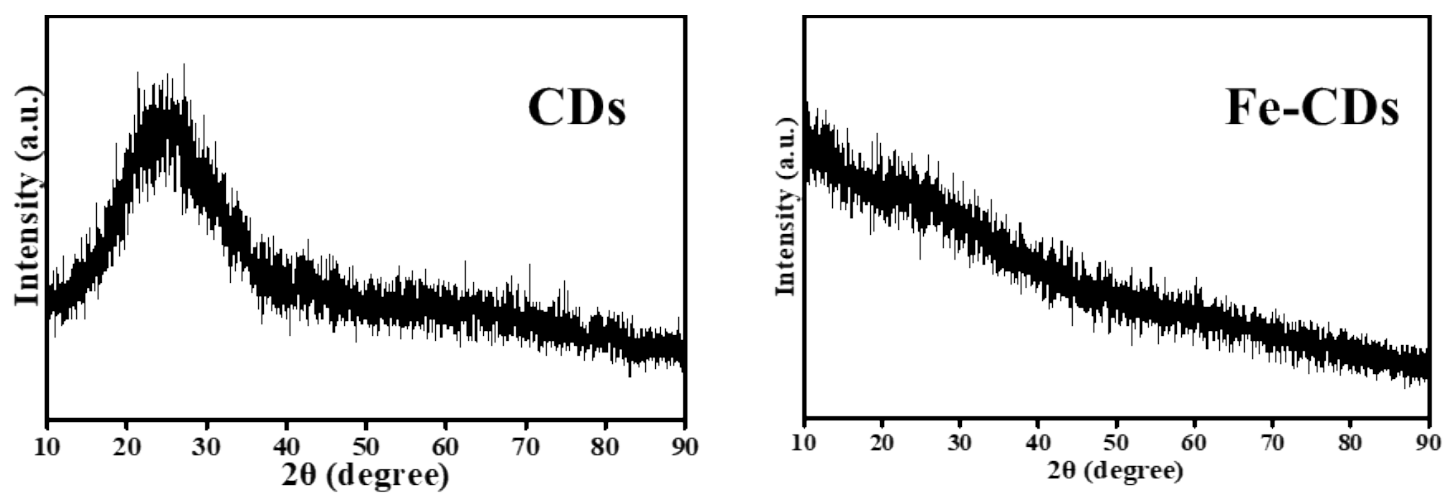

Figure S2. XRD patterns of CDs and Fe-CDs. 


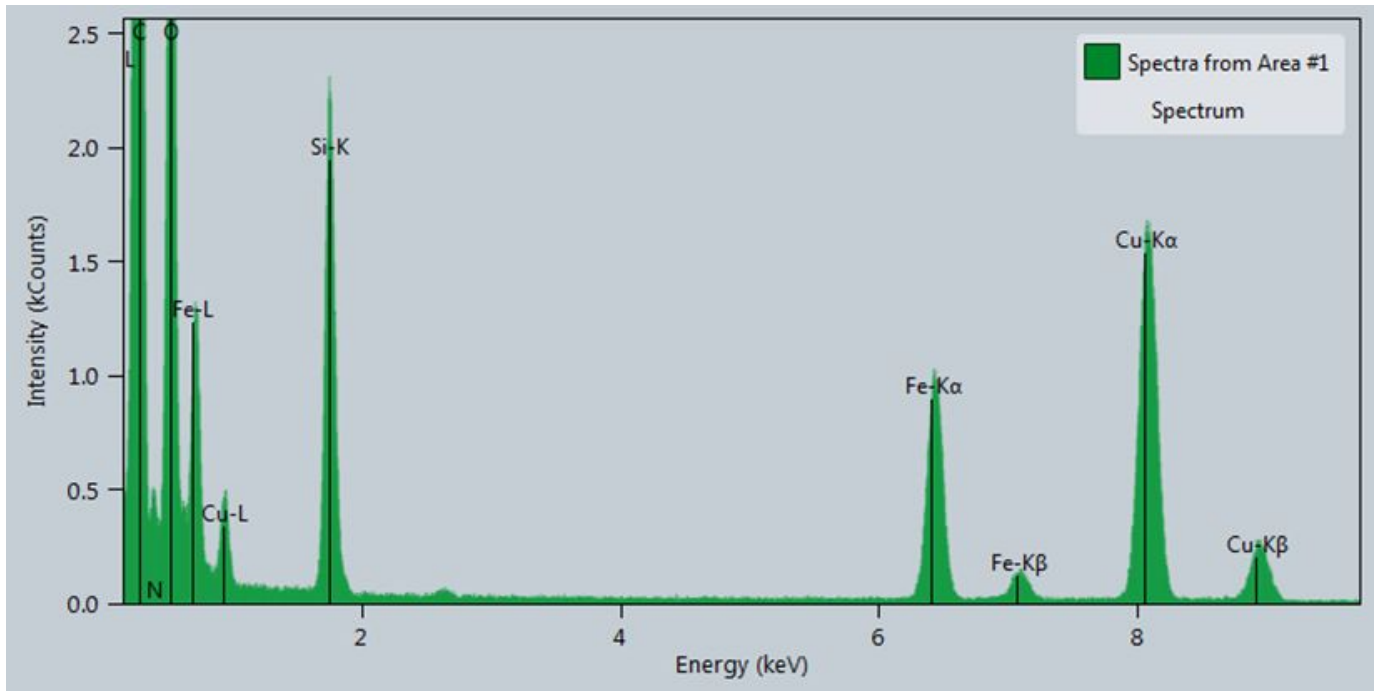

Figure S3. EDAX spectrum of Fe-CDs. 

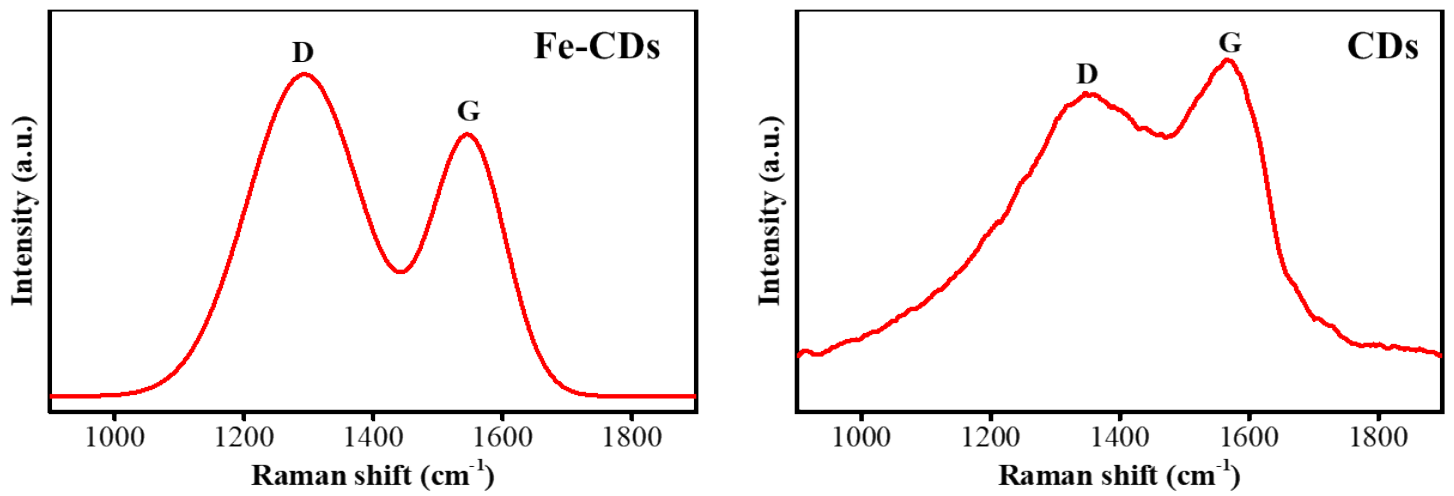

Figure S4. Raman spectra of Fe-CDs and CDs. 

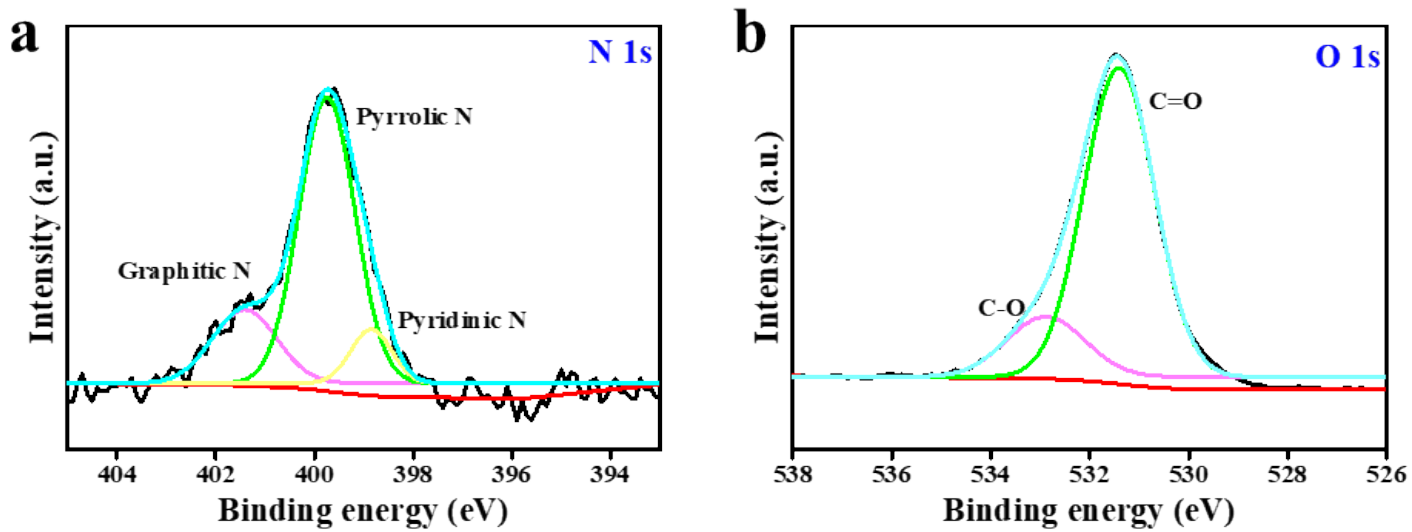

Figure S5. (a) High-resolution XPS N1s spectra and (b) high-resolution XPS O1s spectra of Fe-CDs. 


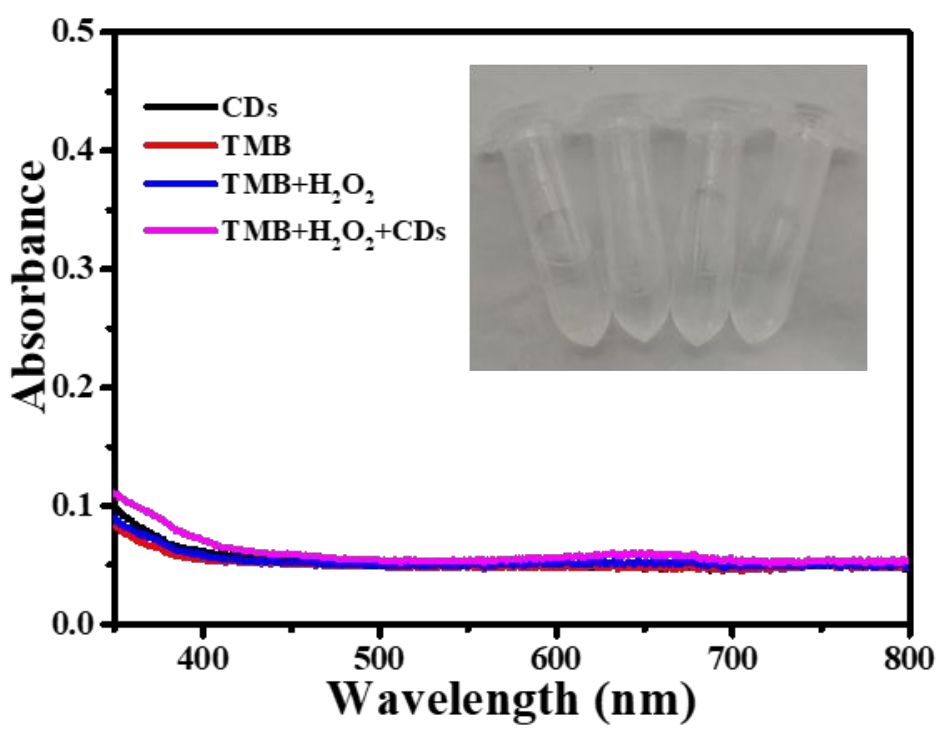

Figure S6. UV-vis absorption spectra of TMB as substrates catalyzed by CDs in the presence of $\mathrm{H}_{2} \mathrm{O}_{2}$. 

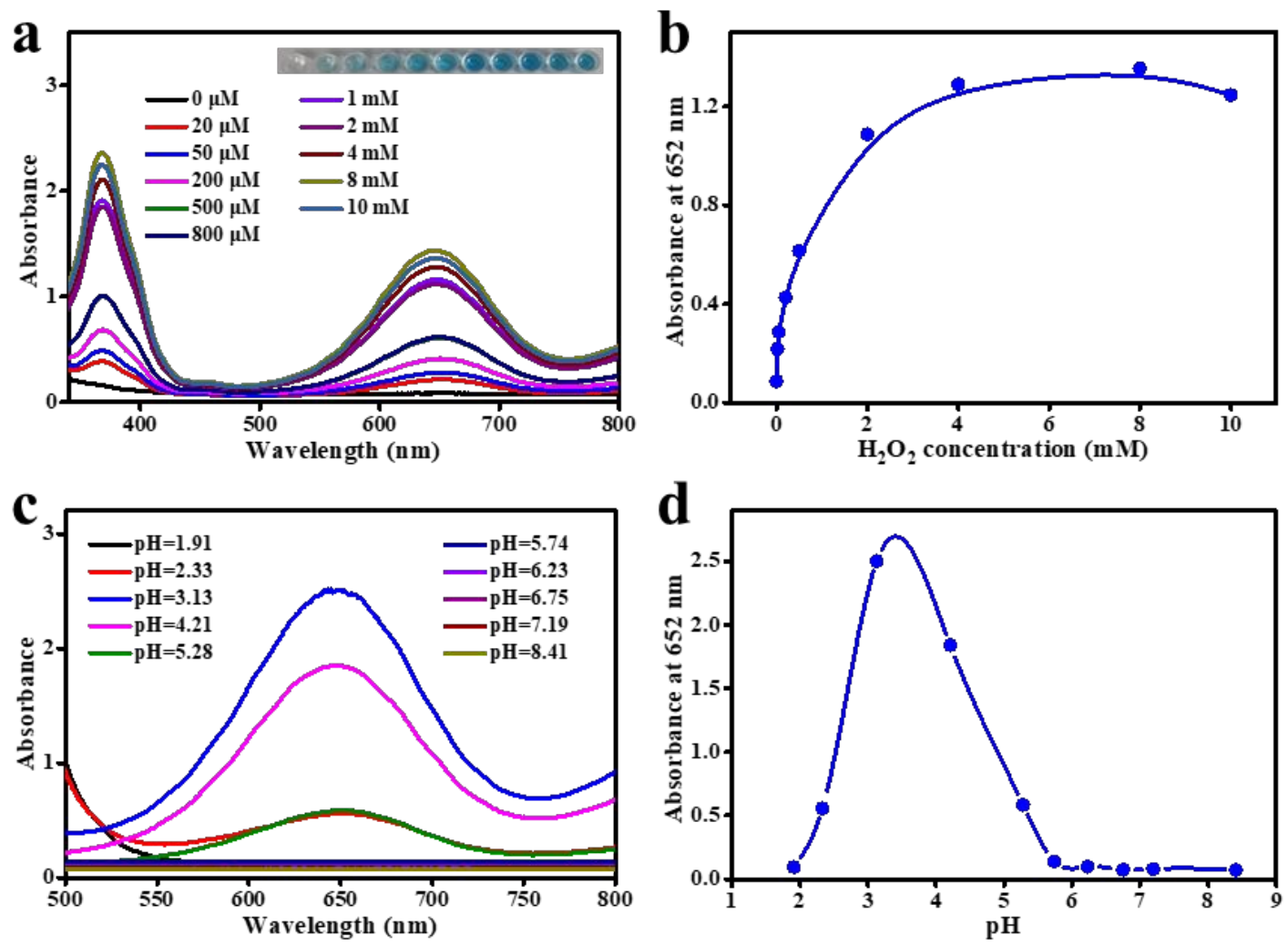

Figure S7. The peroxidase-like activity of Fe-CDs is $\mathrm{pH}$ and $\mathrm{H}_{2} \mathrm{O}_{2}$ concentration dependent. (a) UV-vis absorption spectra of TMB as substrate catalyzed by Fe-CDs with different concentrations of $\mathrm{H}_{2} \mathrm{O}_{2}$. (b) Plot of absorbance at $652 \mathrm{~nm}$ versus concentration of $\mathrm{H}_{2} \mathrm{O}_{2}$. (c) UV-vis absorption spectra of TMB as substrate catalyzed by Fe-CDs at different $\mathrm{pH}$. (d) Plot of absorbance at $652 \mathrm{~nm}$ versus $\mathrm{pH}$. 


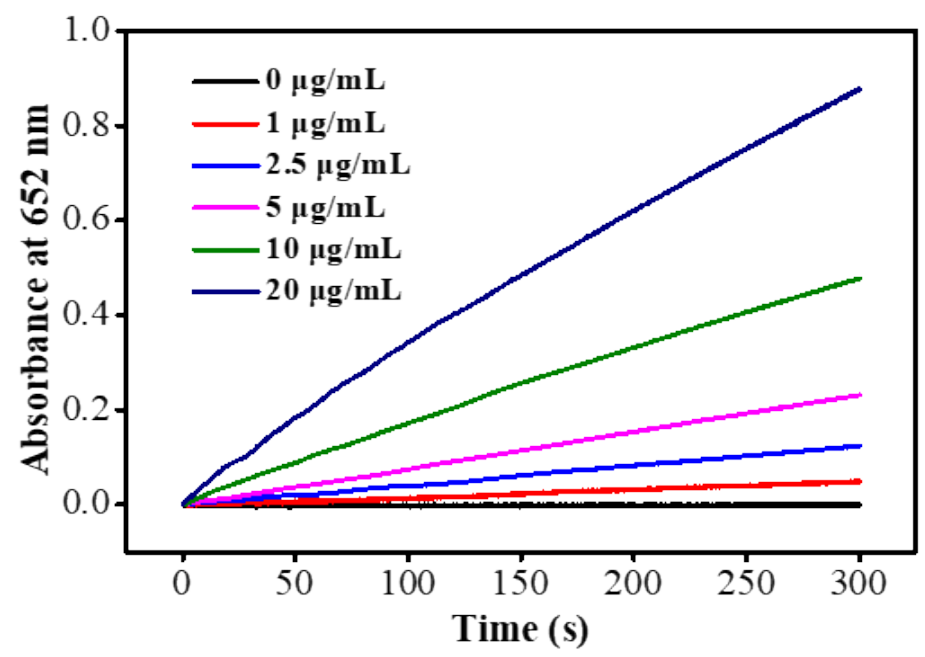

Figure S8. Time-dependent absorbance of oxTMB upon the addition of different concentrations of $\mathrm{Fe}-\mathrm{CDs}$. 


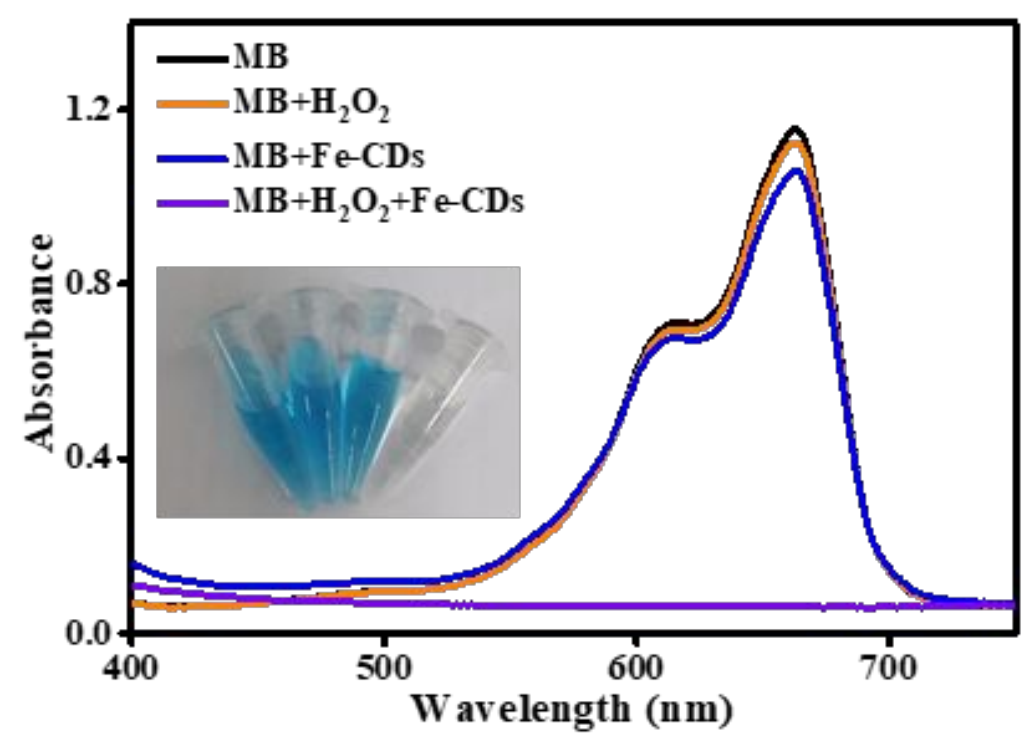

Figure S9. UV-vis absorption spectra of MB as substrate catalyzed by Fe-CDs in the presence of $\mathrm{H}_{2} \mathrm{O}_{2}$. Inset: Corresponding photographs of different reaction conditions. 


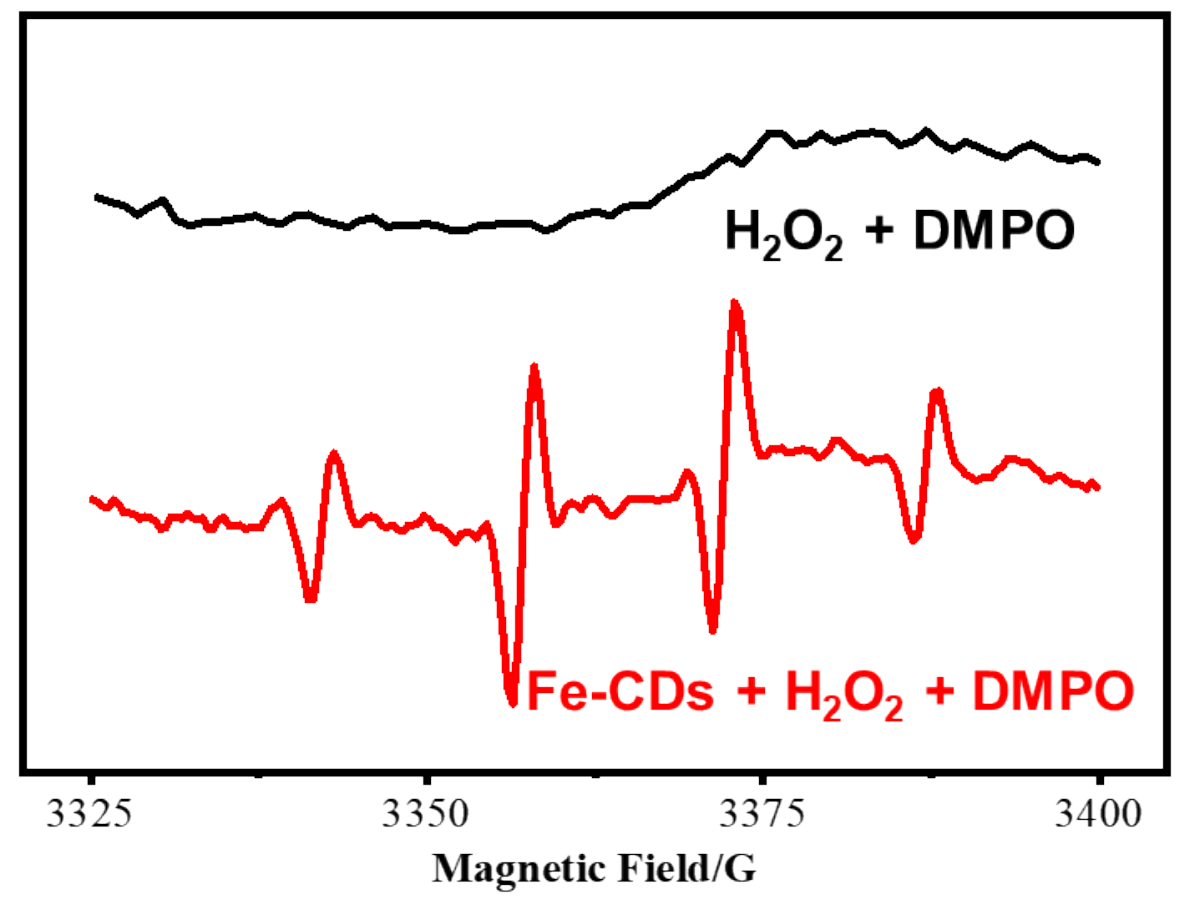

Figure S10. EPR spectra of Fe-CDs/ $\mathrm{H}_{2} \mathrm{O}_{2} /$ DMPO solution and $\mathrm{H}_{2} \mathrm{O}_{2} / D M P O$ solution. 

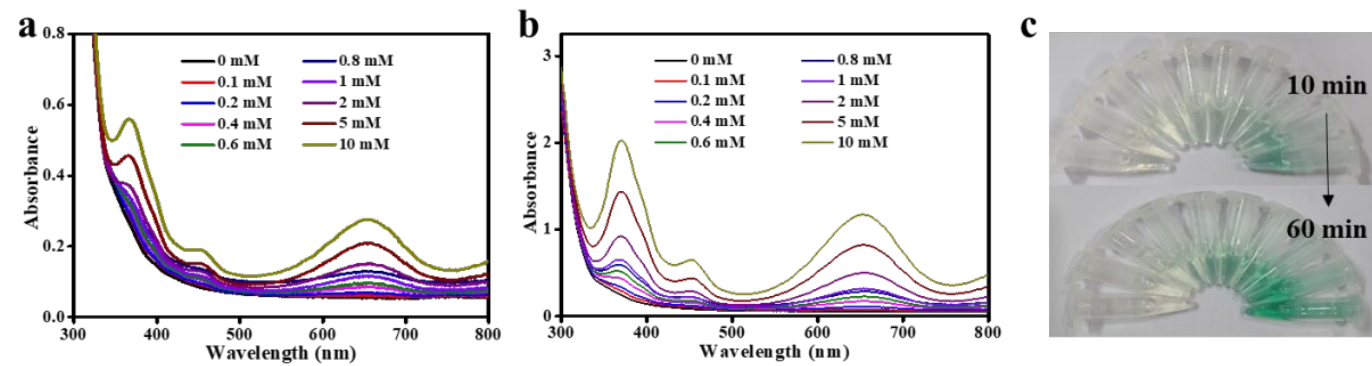

Figure S11. (a\&b) Absorption spectra of reaction systems containing different concentrations of glucose at $10 \mathrm{~min}$ and $60 \mathrm{~min}$, respectively. (c) Corresponding digital pictures of $a \& b$. 

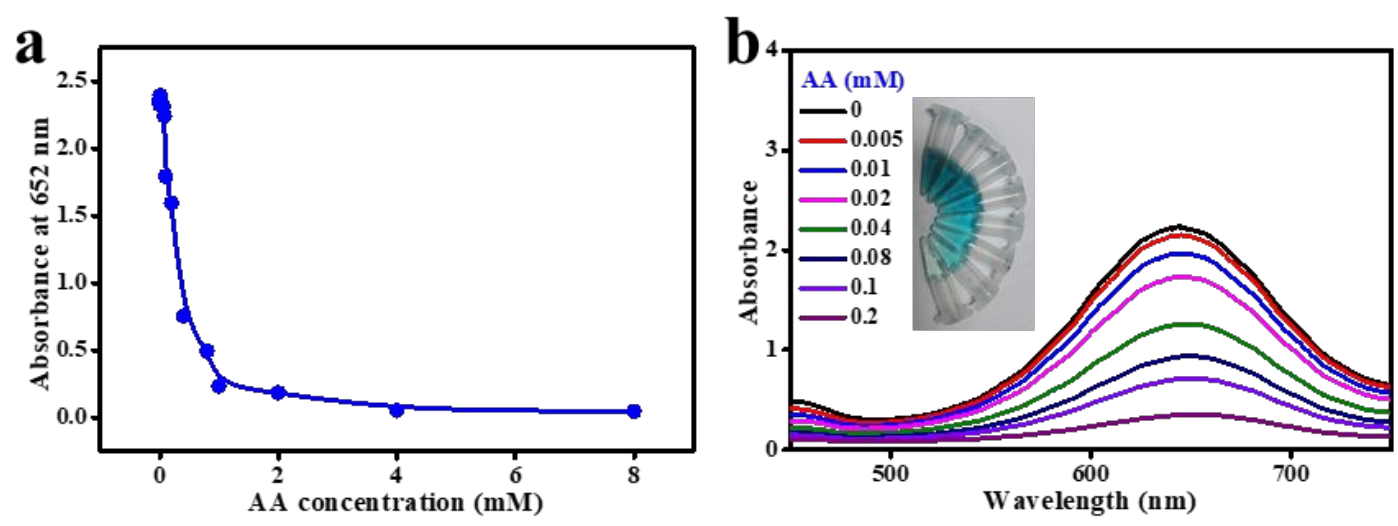

Figure S12. (a) Plot of absorbance at $652 \mathrm{~nm}$ versus concentration of AA. (b) Absorption spectra of reaction systems containing different concentrations of AA. Inset: corresponding digital pictures. 


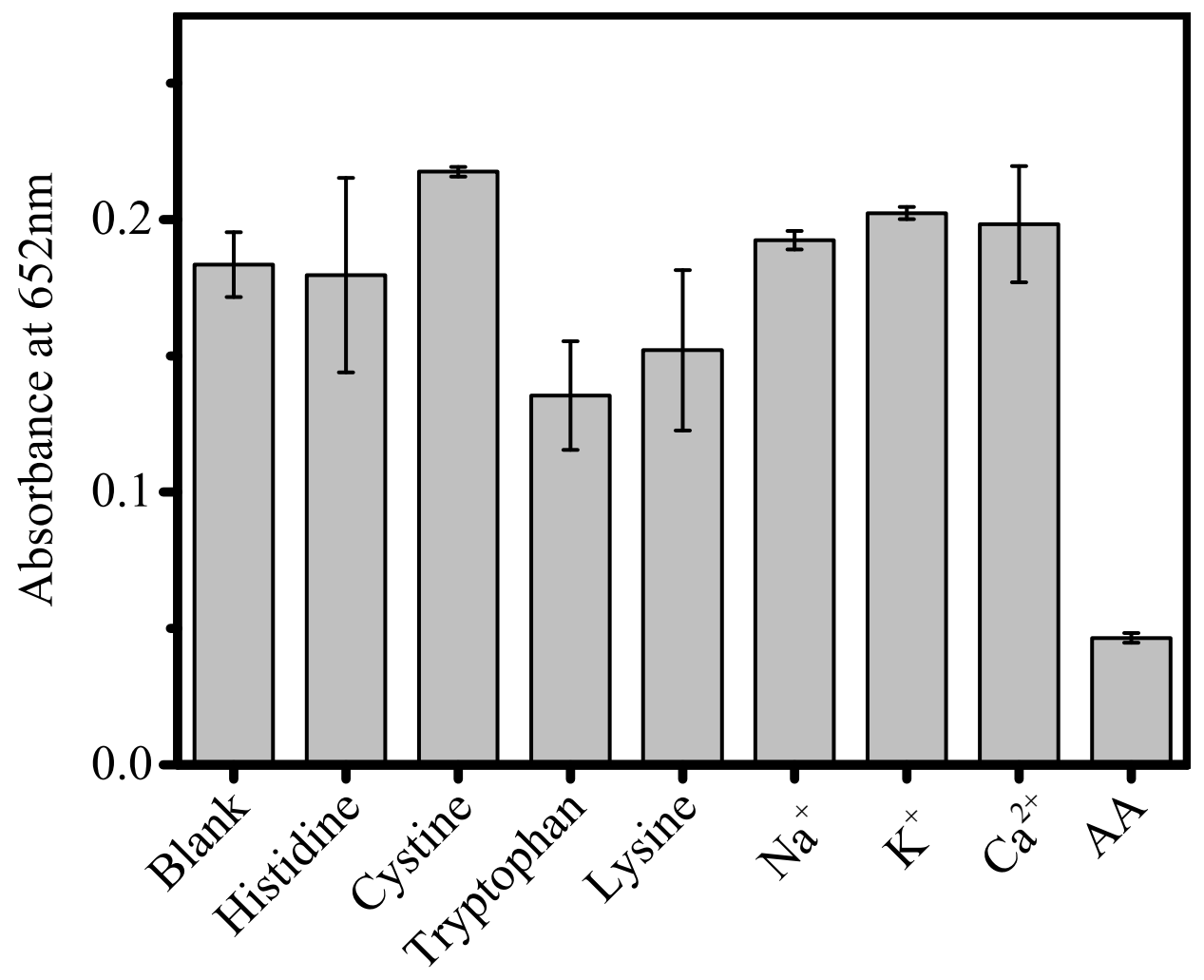

Figure S13. Fe-CDs was used to detect AA, amino acid (Histidine, Cystine, Tryptophan, Lysine), and metal ions $\left(\mathrm{Na}^{+}, \mathrm{K}^{+}, \mathrm{Ca}^{2+}\right)$. 

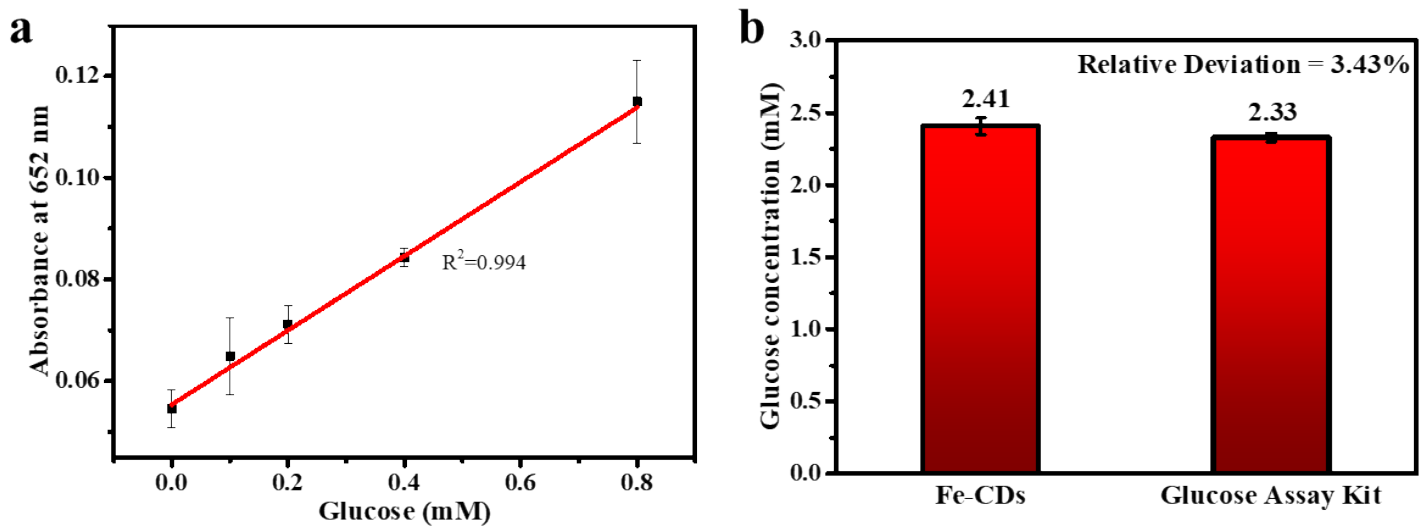

Figure S14. (a) Calibration curve of glucose. (b) Glucose concentration measured by Fe-CDs and Auto Biochemistry analyzer using glucose assay kit. 

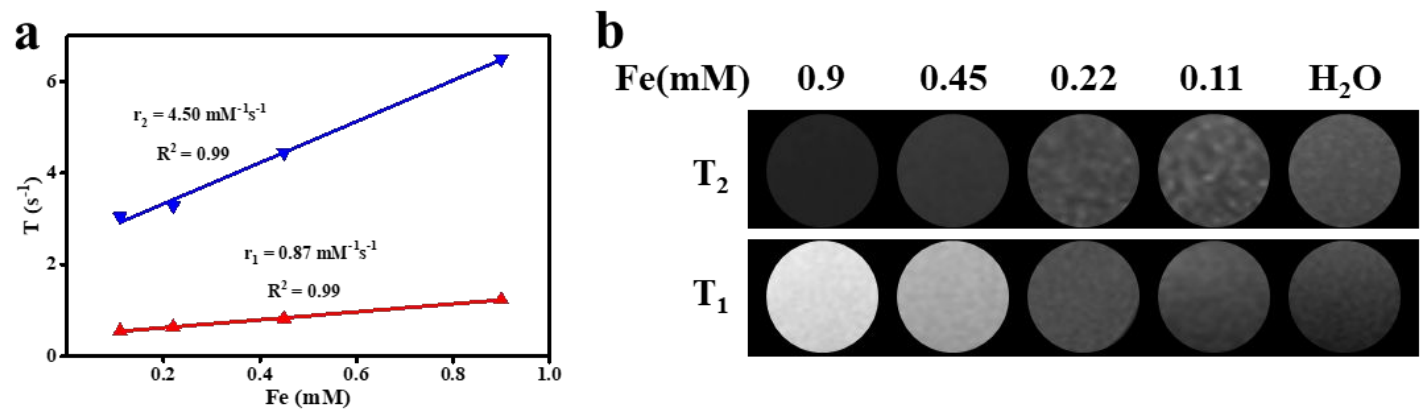

Figure S15. (a) $r_{1}$ and $r_{2}$ relaxivities of Glucose-Fe in aqueous solution investigated on the 9.4T MRI system. (b) $T_{1}$ and $T_{2}$ MR images on the 9.4T MRI system. 

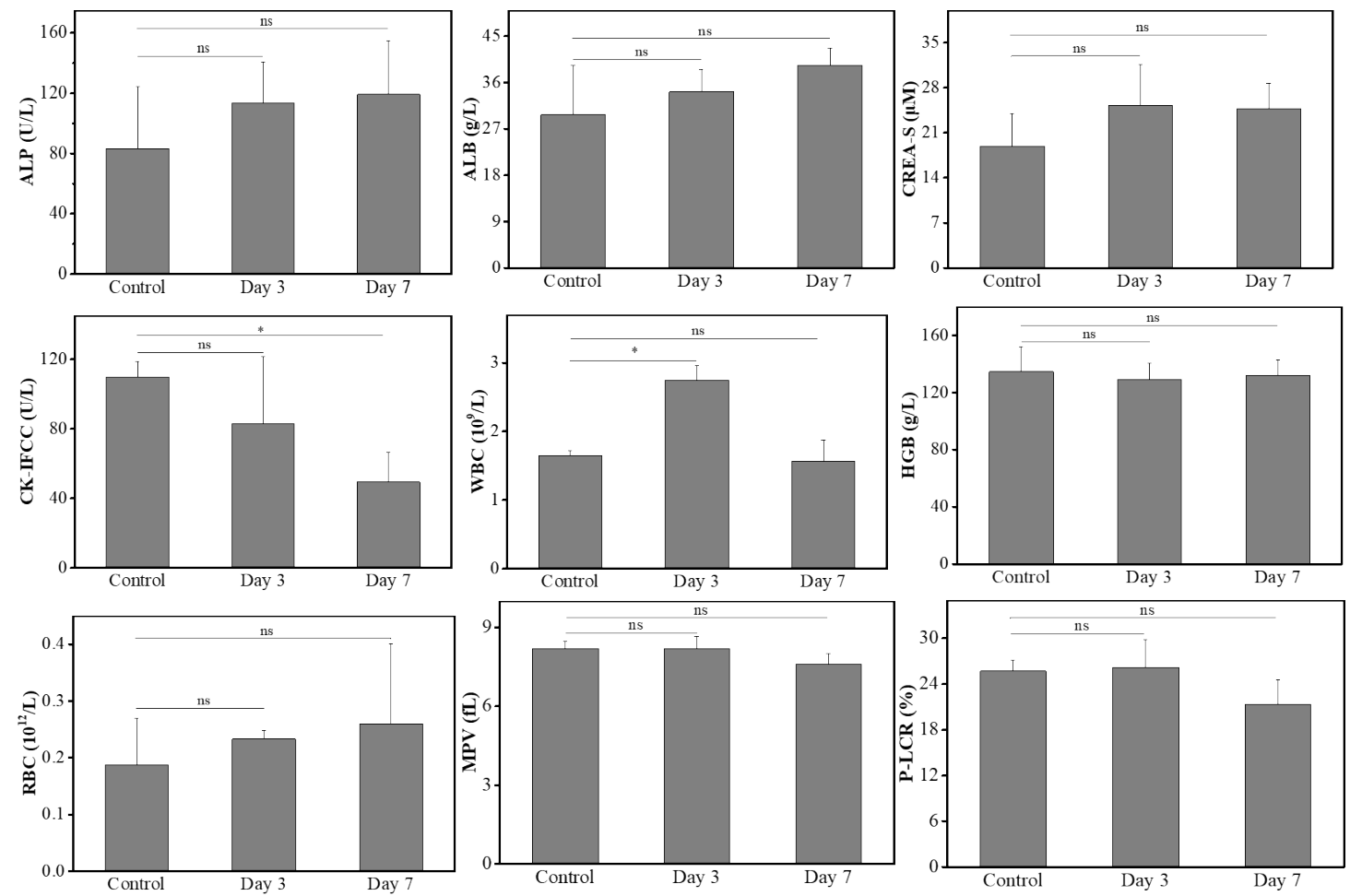

Figure S16. Serum chemistry and hematological analyses of mice treated with Fe-CDs.

Data are mean \pm s.d.. Student's t-test was used to compare the data $(* \mathrm{P}<0.05$, "ns" means no statistical difference). 